\title{
Safety of the transpulmonary ultrasound contrast agent NC100100: a clinical and haemodynamic evaluation in patients with suspected or proved coronary artery disease
}

\author{
Y Myreng, P Mølstad, K Ytre-Arne, M Aas, L Stoksflod, J Ø Nossen, B Oftedal
}

\begin{abstract}
Objective-To evaluate the clinical and haemodynamic safety of NC100100, a new transpulmonary ultrasound contrast agent intended for vascular use.

Design-Pulmonary artery pressures and gas exchange, left ventricular and systemic blood pressure and ECG were measured at baseline, after saline injection, and after each of two increasing doses of NC100100 injected intravenously. Patients-30 patients who were evaluated for suspected coronary artery disease.

Results-No change was detected in any of the haemodynamic variables, or in haematological or clinical chemical parameters. Blood gases were unchanged, as were heart rhythm and arterial oxygen saturation. No serious adverse reactions were reported.

Conclusions-NC100100 appeared to be haemodynamically inert and safe in patients with coronary artery disease.

(Heart 1999;82:333-335)
\end{abstract}

Keywords: NC100100; ultrasound; safety

Ultrasound contrast agents are of particular interest in echocardiography because of their potential use for evaluation of myocardial perfusion. ${ }^{1}$ To obtain such data after intravenous injection, the agents must be able to pass through the lung vasculature to appear in the systemic circulation. ${ }^{2}$ Since ultrasound contrast agents consist of particles containing gas, it is important to ensure that any entrapment in the microvasculature of the lungs and other tissues does not cause adverse effects.

NC100100 is a new ultrasound contrast agent intended for vascular use. The active ingredient is stabilised microbubbles of a perfluorocarbon gas. Preclinical safety assessment shows that the compound is well tolerated, and toxic or adverse effects are seen only at doses much higher than those relevant for clinical use. In dogs NC100100 in doses up to three times the anticipated imaging dose showed no significant haemodynamic effects on the systemic and pulmonary circulation, whereas at doses of 30 and 100 times the anticipated imaging dose there were minor transient cardiovascular effects, probably mediated by histamine release. In monkeys, doses up to 300 times the anticipated imaging dose had no effect on haemodynamic variables. Doses up to 27 times that anticipated to be an imaging dose appeared safe and without any serious adverse effects in healthy volunteers.

Thus NC100100 appears to be haemodynamically safe, and circulatory effects after giving the substance would not be expected in humans. However, it is important to confirm this expectation, and further, to explore whether NC100100 in higher doses than those intended for clinical use might cause any effects on the human pulmonary circulation. Because NC100100 is intended for use in studying myocardial perfusion, both at rest and following stress, it is important that the agent is inert and does not influence circulatory variables. We therefore undertook the present study to investigate haemodynamic effects of NC100100 in patients with coronary artery disease, focusing particularly on the pulmonary circulation.

\section{Methods}

PATIENTS

We studied 30 patients (25 men and five women) with suspected coronary artery disease. Their mean (SD) age was 56 (10) years (range 35 to 74 ), their height was $175(11) \mathrm{cm}$, and body weight was $82(16) \mathrm{kg}$.

Women of child bearing potential and patients with manifest heart failure, with an established diagnosis of pulmonary hypertension, or with unstable cardiac or lung disease were excluded. All patients gave their oral and written informed consent to participate in the study, which was approved by the ethics committee and Norwegian health authorities and performed according to the principles of the Declaration of Helsinki. Medical and angiographic variables are listed in table 1.

Table 1 Medical and angiographic variables among study patients $(n=30)$

\begin{tabular}{ll}
\hline Variable & $n$ \\
\hline Current/previous smoker & $7 / 11$ \\
Angina pectoris & 27 \\
Previous myocardial infarction & 16 \\
Previous coronary angioplasty & 5 \\
Previous coronary artery bypass graft & 4 \\
Diabetes mellitus & 3 \\
Compensated heart failure & 2 \\
Chronic lung disease & 1 \\
Three vessel coronary disease & 13 \\
Two vessel coronary disease & 5 \\
One vessel coronary disease & 7 \\
\hline
\end{tabular}

Accepted for publication 25 March 1999 
Table 2 Clinical chemical and haematological analyses

\begin{tabular}{|c|c|}
\hline Clinical chemistry & Haematology \\
\hline $\begin{array}{l}\text { Sodium } \\
\text { Potassium } \\
\text { Chloride } \\
\text { Calcium } \\
\text { Phosphate } \\
\text { Aspartate aminotransferase } \\
\text { Alanine aminotransferase } \\
\text { Alkaline phosphatase } \\
\gamma \text {-Glutamyl transpeptidase } \\
\text { Bilirubin } \\
\text { Lactate dehydrogenase (total) } \\
\text { Creatine kinase } \\
\text { Uric acid } \\
\text { Urea } \\
\text { Creatinine } \\
\text { Total protein } \\
\text { Albumin } \\
\text { Glucose }\end{array}$ & $\begin{array}{l}\text { Haemoglobin } \\
\text { Packed cell volume } \\
\text { Erythrocyte count } \\
\text { Leucocyte count } \\
\text { Granulocyte count } \\
\text { Lymphocyte count } \\
\text { Eosinophile count } \\
\text { Monocyte count } \\
\text { Thrombocyte count } \\
\text { Reticulocytes (\%) }\end{array}$ \\
\hline
\end{tabular}

INVASIVE PROCEDURE AND MEASUREMENTS

A Swan-Ganz CCOmbo thermodilution catheter (Baxter Healthcare Corporation, Irvine, California, USA) was advanced from the femoral vein into the pulmonary circulation and connected to a standard pressure transducer for continuous monitoring of pulmonary arterial pressure and measurement of the pulmonary capillary wedge pressure (PCWP) by inflation of the distal balloon. Cardiac output was measured by the Vigilance Computer System (Baxter). ${ }^{34}$

Femoral arterial pressure was monitored continuously throughout the procedure, as were cardiac rhythm and arterial oxygen saturation (pulse oximetry). At the beginning and end of the invasive procedure, left ventricular pressure was recorded through a $6 \mathrm{~F}$ fluid filled pigtail catheter.

Each patient received three intravenous injections through an $18 \mathrm{G}$ Venflon cannula into a large antecubital or cubital vein. First, saline $0.10 \mathrm{ml} / \mathrm{kg}$ was injected. Then two injections of NC100100 (5 $\mu \mathrm{l}$ microbubbles $/ \mathrm{ml}$ ) $0.02 \mathrm{ml} / \mathrm{kg}$ and $0.10 \mathrm{ml} / \mathrm{kg}$ (corresponding to $0.1 \mu \mathrm{l}$ and $0.5 \mu \mathrm{l}$ perfluorocarbon gas $/ \mathrm{kg}$, respectively) were given. The injection rate was $1 \mathrm{ml} / \mathrm{s}$, and each injection was followed by a flush of $10 \mathrm{ml}$ saline.

Pulmonary artery pressure, cardiac output, systemic arterial pressure, heart rate, and arterial oxygen saturation were measured at baseline, after the saline injection, and one, three, and five minutes after each of two injections of NC100100. Between the injections at least 10 minutes were allowed for stabilisation, and the second injection of NC100100 was given only after pulmonary artery pressure had returned to baseline. In addition, arterial blood gas analyses were performed at baseline, five minutes after saline injection, and five minutes after each of the test substance injections.

HAEMATOLOGICAL AND CLINICAL CHEMICAL ANALYSES, URINE ANALYSIS, ECG, AND CLINICAL OBSERVATIONS

Blood samples were drawn at baseline, $10 \mathrm{~min}-$ utes after the last injection, and on the day after the procedure. They were analysed for the haematological and clinical chemical variables listed in table 2. In addition, coagulation factors in the external system were tested by the prothrombin time. Urine samples were analysed for the presence of glucose, blood, and protein by sticks at baseline and on the morning the day after the procedure. A 12 channel ECG was recorded at baseline, immediately after the procedure, and on the following day. The patients were instructed to report any discomfort or sensation during the 24 hours after the procedure. Adverse events were recorded and coded according to the WHO Adverse Reaction Dictionary.

\section{STATISTICAL METHODS}

Continuous variables were compared with paired $t$ test. All values are given as mean (SD), and the significance level was $\mathrm{p}<0.05$.

\section{Results}

Haemodynamic variables obtained at right heart catheterisation, the results of arterial pressure monitoring, heart rate, and the oxygen saturation in the arterial blood are summarised in table 3. All variables were within normal ranges at baseline, and there was no statistically significant change in any value after saline injection, nor after any of the two increasing doses of NC100100. There was no change in left ventricular end diastolic pressure from baseline to the end of the procedure (14 (4) v 15 (3) $\mathrm{mm} \mathrm{Hg}$ ), nor in peak positive left ventricular $\mathrm{dP} / \mathrm{dt}$ (1543 (339) v 1592 (343) $\mathrm{mm} \mathrm{Hg} / \mathrm{s}$ ) or peak negative left ventricular $\mathrm{dP} / \mathrm{dt}(1583$ (587) v 1798 (479) $\mathrm{mm} \mathrm{Hg} / \mathrm{s})$. In addition, no changes were observed in arterial blood gases or ECG.

The haematological and clinical chemical values were all within the reference range at all three measurement times, except for glucose

Table 3 Haemodynamic variables

\begin{tabular}{|c|c|c|c|c|c|c|c|c|}
\hline \multirow[b]{2}{*}{ Variable } & \multirow[b]{2}{*}{ Baseline } & \multirow[b]{2}{*}{ Saline } & \multicolumn{3}{|c|}{ NC100100-low dose } & \multicolumn{3}{|c|}{ NC100100_high dose } \\
\hline & & & $1 \mathrm{Min}$ & $3 \mathrm{Min}$ & $5 \mathrm{Min}$ & $1 \mathrm{Min}$ & $13 \mathrm{Min}$ & $5 \mathrm{Min}$ \\
\hline Systolic PAP (mm Hg) & $26(7)$ & $27(7)$ & $28(7)$ & $27(7)$ & $27(7)$ & $27(7)$ & $28(7)$ & $27(7)$ \\
\hline Diastolic PAP ( $\mathrm{mm} \mathrm{Hg}$ ) & $13(5)$ & $13(5)$ & $13(5)$ & $13(5)$ & $12(5)$ & $14(6)$ & $13(5)$ & $13(5)$ \\
\hline Mean PAP $(\mathrm{mm} \mathrm{Hg})$ & $18(6)$ & $19(5)$ & $19(5)$ & $18(5)$ & $18(6)$ & $19(6)$ & $18(5)$ & $18(5)$ \\
\hline PCWP (mm Hg) & $12(5)$ & $12(5)$ & $12(5)$ & $11(5)$ & $11(5)$ & $12(5)$ & $12(5)$ & $12(5)$ \\
\hline PVR (dyn.s.cm ${ }^{-5}$ ) & $1.2(0.6)$ & $1.2(0.4)$ & $1.2(0.6)$ & $1.3(0.6)$ & $1.3(0.5)$ & $1.4(0.5)$ & $1.2(0.6)$ & $1.3(0.4)$ \\
\hline Systolic BP (mm Hg) & $138(23)$ & $141(22)$ & $141(22)$ & $140(23)$ & $140(23)$ & $143(24)$ & $141(22)$ & $142(23)$ \\
\hline Diastolic BP (mm Hg) & $72(12)$ & $72(12)$ & $72(11)$ & $71(11)$ & $71(11)$ & $73(12)$ & $72(11)$ & $72(11)$ \\
\hline Cardiac output $(1 / \mathrm{min})$ & $5.7(1.6)$ & $5.7(1.6)$ & $5.5(1.6)$ & $5.4(1.7)$ & $5.4(1.7)$ & $5.4(1.6)$ & $5.4(1.6)$ & $5.4(1.5)$ \\
\hline Heart rate (beats $/ \mathrm{min}$ ) & $59(9)$ & $58(9)$ & $57(8)$ & $57(9)$ & $57(9)$ & $59(8)$ & $58(9)$ & $58(9)$ \\
\hline $\mathrm{SaO}_{2}(\%)$ & $96(1.2)$ & $97(1.1)$ & $97(1.1)$ & $97(0.9)$ & $97(0.9)$ & $96(1.3)$ & $96(1.5)$ & $96(1.2)$ \\
\hline
\end{tabular}

Values are mean (SD).

$\mathrm{BP}$, blood pressure; PAP, pulmonary artery pressure; PCWP, pulmonary capillary wedge pressure; PVR, pulmonary vascular resistance; SaO ${ }_{2}$, oxygen saturation in arterial blood. 
which was slightly raised $(6.8 \mathrm{mmol} / \mathrm{l})$ the day after the procedure, presumably because of lack of fasting at the time of blood sampling. There was no statistically significant change from baseline in any variable.

There were no deaths or serious adverse events reported within the trial period. Six patients experienced a total of 11 adverse events, all of which classified as mild. Seven of the events reported were judged to be unrelated to the study drug, whereas the relation to the study drug was uncertain for four events. These were chest pain for five minutes after injection of the lower dose of NC100100 and palpitations for one hour seven hours after the injection in one patient; diarrhoea starting eight hours after and lasting until the next day in one patient; and nausea for 30 minutes immediately after the last injection in one patient. The seven adverse events-classified as such, but judged as unrelated to the drugwere back pain (from lying on the investigating table) in two patients, hypoxia (slight and fluctuating, present before and during the study) in two, tremor (nervous reaction) in one, chest pain (non-specific, present before the study) in one, and hyperglycaemia (which appeared to be from undiagnosed diabetes) in one.

\section{Discussion}

The gas filled microbubbles which constitute the new ultrasound contrast agent NC100100 are smaller than the diameter of capillaries. It is therefore unlikely that they would cause embolisation in the microvasculature of the lungs and other tissues. However, injection of particulate substances into the circulation might trigger responses such as histamine release and activation of vasoactive proteins, at least in certain species. ${ }^{5}$ It is therefore promising for the future clinical use of NC100100 that our main finding was that it appeared to be haemodynamically inert with no sign of interference with the pulmonary circulation. Furthermore, it was subjectively well tolerated and had negligible side effects. There was no interference with pulmonary gas exchange. These findings are in concordance with those of a similar patient study on Albunex, a contrast agent consisting of air filled albumin microspheres, ${ }^{6}$ as well as an experimental study in dogs using FS-069, ${ }^{7}$ which, like NC100100, is a second generation ultrasound contrast agent.

The same beneficial properties were also present with respect to the systemic circulation. Any adverse effects on the cardiac microcirculation might have been reflected in increased left ventricular filling pressures, or in changes in positive and negative left ventricular pressure development, or both. Owing to the restrictions set in the protocol, such recordings were obtained at baseline and at the end of the procedure only. However, NC100100 has a relatively long half life and hence circulates for several minutes after injection. This fact strongly suggests that any untoward effect on left ventricular haemodynamics would have been detected in the recordings at the end of the procedure, as those were influenced by the whole burden of contrast throughout the experiment.

We chose to perform the study in a population of patients with suspected or proved coronary artery disease because these patients are considered at present to be the target group for the new ultrasound contrast agent. It is promising that the patients did well and tolerated the substance, even those with rather advanced coronary artery disease. As the study was for phase II safety documentation, certain restrictions had to be taken into account in the protocol. Thus we have not acquired data from unstable patients or patients with raised pulmonary artery pressure. Also, the effect of larger and cumulative doses was beyond the scope of this early study. It is possible that ultrasound might increase the rate of destruction of the microbubbles, but unlikely that this would in any way alter the safety of the product documented in the present study. Echocardiography was not routinely performed, as the patients had to be studied supine during the catheterisation. Although ultrasound imaging was not a part of the protocol, contrast passage through the lungs was verified in a few selected patients. Thus we have no data on the efficacy of NC100100, which is, however, currently being investigated in several specially designed trials.

1 Rovai D, Monaghan MJ. New contrast agents and technologies for myocardial contrast echocardiography. Heart gies for myocardial cont

2 Feinstein SB, Cheirif J, Ten Cate FJ, et al. Safety and efficacy of a new transpulmonary contrast agent: initial multicenter results. . Am Coll Cardiol 1990;16:316-24.

3 Yelderman M. Continuous measurement of cardiac output with the use of stochastic system identification techniques. f Clin Monit 1990;6:322-32.

4 Munro HM, Wood CE, Taylor BL, et al. Continuous invasive cardiac output monitoring - the Baxter/Edwards Critical-Care Swan-Ganz IntelliCath and Vigilance system. Clin Intensive Care 1994;5:52-5.

5 Ostensen J, Hede R, Myreng Y, et al. Intravenous injection of Albunex microspheres causes thromboxane mediated pulmonary hypertension in pigs, but not in monkeys or rabbits. Acta Physiol Scand 1992;144:307-15.

6 Geny B, Mettauer B, Muan B, et al. Safety and efficacy of a new transpulmonary echo contrast agent in echocardiographic studies in patients. $f$ Am Coll Cardiol 1993;22: graphic stud $1193-8$.

7 Skyba DM, Camarano G, Goodman NC, et al. Hemodynamic characteristics, myocardial kinetics and microvascular rheology of FS-069, a second-generation echocardiographic contrast agent capable of producing myocardial opacification from a venous injection. $7 \mathrm{Am}$ Coll Cardiol 1996;28:1292-300. 